20

\title{
OST - An Object-Oriented Computer Networks System Specification Tool
}

Claudia Maria Sbardelotto, Lisandro Zambenedetti Granville, Luciano Paschoal Gaspary, Maria Janilce B. Almeida

Federal University of Rio Grande do Sul (UFRGS-CPGCC)

Campus do Vale, Bloco IV - Bento Gonçalves, 9500 Agronomia - PO 15064, ZIP 91501-970, Brazil-Tel: +55.51.316.6161, Fax: +55.51 .319 .1576$

\{claudias, granvile, paschoal, janilce\}@inf.ufrgs.br

\begin{abstract}
With the purpose of lessening the software production efforts in computer networks area, the use of tools that help the system specification and implementation process is needed. This work presents a development tool based on the NSOMA methodology, intended to help the designers in the formal definition of specifications. It is composed of a graphical editor, an animation module that allows the visualization of specification dynamic behavior and a source code automatic generating module.
\end{abstract}

\section{Keywords}

computer networks, specification tool, NSOMA, object orientation, animation, source code generation 


\section{INTRODUCTION}

The use of tools for developing software is currently a fact, due to advantages such as reduced production time and easy system maintenance, documentation and expansion. The use of a number of features available for each tool provides help for the various stages of software developing.

There are many system specification and implementation environments designed for computer networks. Most designers, though, still use primitive, userunfriendly methodology, as these environments are usually based on complex Formal Description Techniques (FDTs) (Cohen, 1986). An Object Orient Specification Tool (OST) has been developed at Federal University of Rio Grande do Sul bearing this difficulty in mind. OST associates a modeling and developing methodology to a formal specification technique. This tool allows better comprehension and organization of the system components and thus becomes a very efficient way to produce easy-to-use tools regardless of the programming language they are based on, whieh are only a few and mastered by few designers.

OST is based on the Network System Object Modeling Approach (NSOMA) (see item 2 below), integrating object-orientation concepts to SDL (Specification and Description Language). OST is composed of three modules: a graphic editor, which allows creating and editing a specification from NSOMA's graphic grammar, an animation module, which provides instruments to visualize the dynamic behavior of the specifications, and an automatic source code generator. These modules are presented in sections 3, 4 and 5, respectively and section 6 introduces an example.

\section{NETWORK SYSTEM OBJECT MODELING APPROACH (NSOMA)}

The goal of NSOMA is to make SDL easier to use, associating it to the objectorientation paradigm under the Object Modeling Technique (OMT) method (Almeida, 1994) (Rumbaugh, 1991). As the human mind can see the world as a series of inter-related objects, the use of this concept furthers comprehension. Thus, the real world is brought closer to the computing world. It also allows the possibility of reusing previously developed entities in the creation of new software.

Because NSOMA specifications are hierarchically defined, this approach is broken into three specification levels. These levels allow the designers to start from a more abstract level and then refine their specification, bringing it closer to the implementation. 
The following are NSOMA specification levels:

- Abstract structural level: at this level, classes' behavior and relations are determined.

- Detailed structural level: at this level, each class's behavior is determined. Ports, internal signals, attributes and operations are defined.

- Operation level: at this level, each previously defined operation is detailed.

The abstract structural level description is based on OMT concepts. It allows the definition of highly abstract classes that form a network system and the relations between them. Their corresponding objects represent elements of a network system - protocols, functions, services, entities. These classes are described and identified by their type, name, attributes and operations.

A class can be defined from another's definition. In such case, one says the first inherits from the second and calls it a subclass, which may inherit attributes and operations from the parent class totally or partially. In addition, a class can be defined as a combination of other classes, though preventing the latter from inheriting attributes or operations from the former. A class can inherit classes that were defined by combination. In such case, the subclass inherits all classes that compose the parent class.

The detailed structural level is where attributes, operations and internal signals are actually defined for a given class. Every class modeled at the abstract structural level is refined at the detailed structural level. The internal signals allow communication between operations of the same class. They can be synchronous or asynchronous.

Ports and external signals supply communication between elements of different classes. It can also be synchronous or asynchronous. Communication is considered synchronous when the source operation enters a hold state for a response signal, thus being blocked indefinitely, whereas asynchronous communication does not hold and therefore is not blocked. External signals are defined outside classes' behavior, which allows their further individual use.

The operation level allows every previously defined operation to have its behavior diagramed, thus determining the system's control flux. Hierarchically, it is the closest level to implementation and it is strongly based on SDL. Graphically, this level is described by diagrams that represent a state machine of the operations.

\section{THE GRAPHIC EDITOR}

This editor implements all functions necessary to system specification in NSOMA. The software uses NSOMA's graphic syntax to help the user interact more naturally. Specifications are created through the manipulation of the 
grammar graphics (Gaspary, 1995) (Granville, 1995). The textual approach is not neglected, though, allowing the user to textually visualize the specifications being



built. Figure 1 shows the tool's main interface.

Figure 1 OST's graphic interface

From the File menu, the user can create new specifications, load preexistent specifications from a database, save specifications onto database as well as retrieve classes from a class library. From the Edit menu, objects can be selected from the editor and copied to Windows transfer area, which can prove helpful to documentation using other software. The $A S L, D S L$, $O L$ and $E S$ menus are used to select symbols corresponding to each of NSOMA's levels. Animation and Generate Source Code start operations from their respective modules, which are presented below. Finally, Window and Help have window management functions for OST, which are standard to Windows applications.

The upper toolbar provides quick access to the most commonly used functions. It accesses these functions: creation of a new specification, loading and saving an ongoing specification, deletion of selected symbols and their copy to Windows transfer area, reading the external signals diagram and help.

To start a new specification, select New from the File menu or click on the corresponding icon on the toolbar. That will open a window representing the abstract specification level. On the upper left corner the 
words Key and Describer can be seen, without any links to text. Also, four buttons on the left become active. These are the functions that can be used at this level. To create a new class, for instance, click on the class button (1) of figure 1. Next, use the mouse to insert new classes in the window. Initially, the application will automatically generate names and types for the classes in order to avoid repetitions.

To include a class from the library in your specification, select Load class from the File menu. A dialog box will display all library classes so that one can be picked. The chosen class is then loaded from the database and included in the diagram.

Buttons (3) and (4) on figure 1 allow the representation of class relations. Click on the corresponding button and then on a class graphic to create a line that represents a relation between two classes.

Finally, button (2) brings the user back to selection mode. Using this, the user can move the graphics on the screen by dragging them. Also, many objects can be selected simultaneously and then moved, copied to the transfer area or deleted.

Figure 2 shows the active menu for a class Class 3 . This class can have its name and type changed, as well as some graphic features of inheritance and composition.

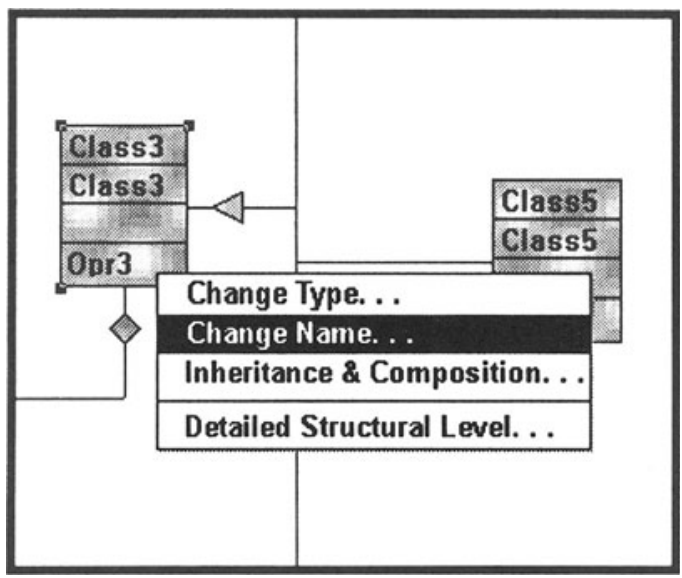

Figure 2 Context-sensitive menus

The last option of the menu starts edition at the detailed structural level. Double clicking on a class also starts this function.

Accessing the detailed structural level opens a new window. New buttons are activated on the left, disabling the buttons for the previous 
level. This new level enables functions for creating operations, internal signals (7), external signals (8) and attributes (6), shown in figure 1 . The manipulation of objects follows the same pattern for manipulation as the abstract level.

NSOMA's last level is accessed by double-clicking an operation, the same pattern used to access the detailed level. Once again, the left-hand toolbar is updated to show the options valid for the current operational level, as shown in figure 1.

At any level, a specification's textual description can be read. To do that, select this option from the menu in the corresponding level. The text is presented in a new dialog box. This cannot be altered, though, in order to assure consistency with the graphic specification.

To include all these functions in the editor, an object-oriented programming approach was used to build it. The basic classes that were implemented can be divided into two separate groups, database class and graphic class (Trainini, 1995). The database classes (Data_ob) are responsible for the semantic consistency of a given specification (see figure 3). Each new element added to the diagram is actually a database object. If it cannot be created, some database rule violation can be assumed. Further information on how database elements interrelate to keep specification consistency can be found in (Gaspary, 1995) (Granville, 1995).

On the other hand, the graphic classes are responsible for the implementation of all graphic functionality. There are basically a window class (TDragDropWindow) and a graphic object class (TShape). These two primitive classes work together to implement drag-and-drop and multiple selection functions, among others. Window classes for each specification level were derived from TDragDropWindow. All graphic symbols used in the editor were derived from TShape.

The basic classes are divided into two separate groups for the sake of comprehension. Actually, there is high interaction between the two groups. In fact, any graphic element is a database object and a graphic object at the same time. It must be consistent to its specification as well as have all the necessary graphic functionality.

The basic classes, in their turn, are also derived from a library set (OWL, which accompanies Borland $\mathrm{C}++)$. OWL contains many of the required features. Figure 3 shows a general map of the classes used by the editor. 


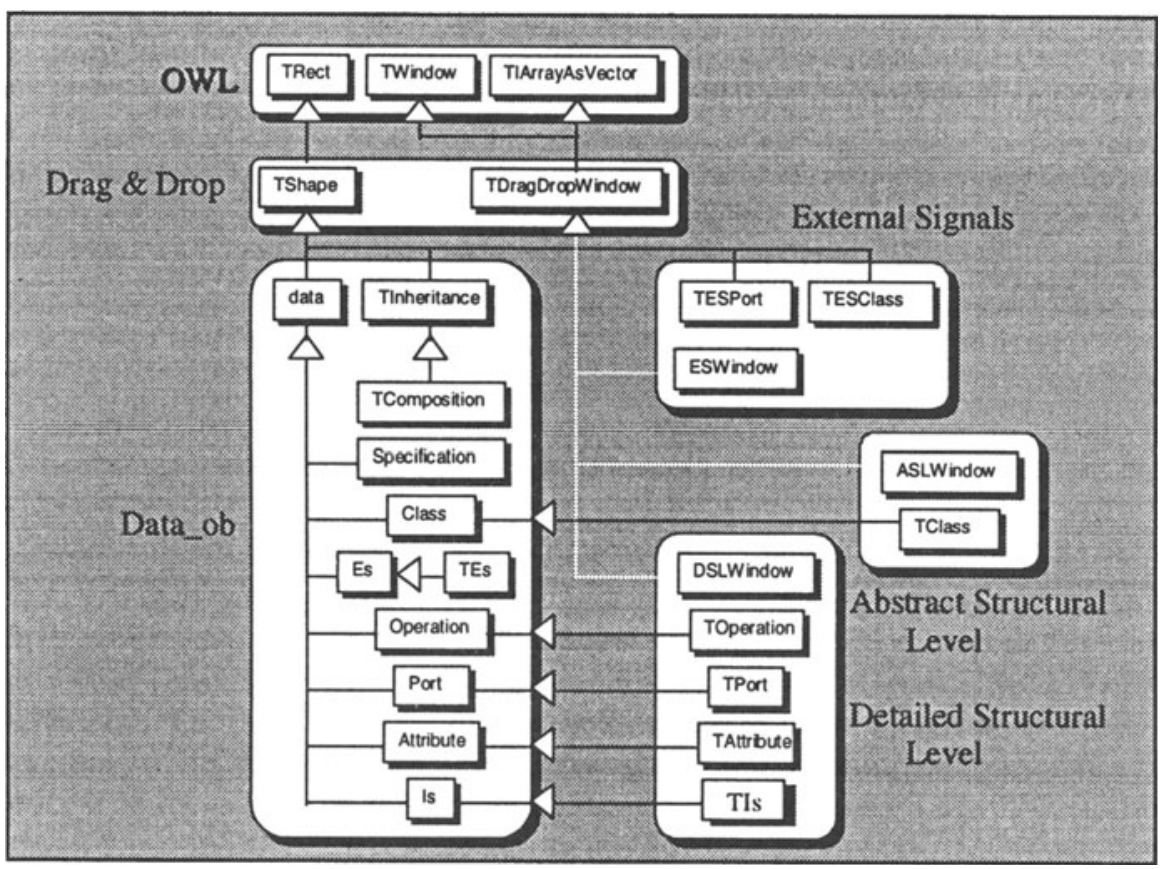

Figure 3 Set of classes for the OST editor

\section{ANIMATION MODULE}

The animation of a system's dynamic behavior is a basic need to its definition and specification (Allende, 1995). It fosters the understanding of the description being specified, translated as reduced construction cycles since a number of problems can be verified during the early development stages (Verilog, 1994).

OST provides an environment to visualize animation, in addition to plenty of statistic data and some deadlock-detection facilities. This kind of functionality can be found in verification systems.

The animation was implemented using, basically, symbols from the operational level and operation representation structure (Gaspary, 1996). The animation controls were implemented in two new classes called AnimOL_Sym and AnimOperation, which relate to the editor's structure as seen in figure 4 . AnimOL_Sym associates every animation-relevant symbol to a count variable, in order to provide statistics on the use of this symbol. Also, it has a drawing method for the symbol being run, so that it can be highlighted. AnimOperation implements an input signal queue for each operation, which keeps the signals 
from being lost. Using the same pattern as for symbols at the operational level, a drawing method for the highlighted operation symbol should follow.

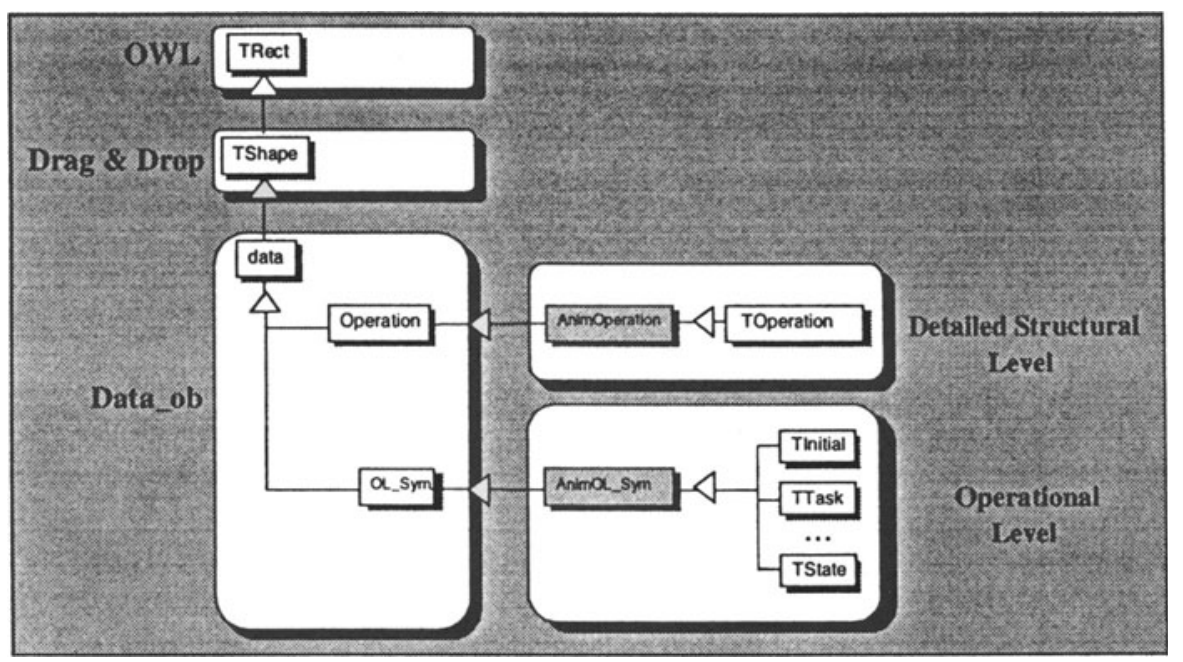

Figure 4 AnimOL_Sym and AnimOperation classes

Figure 5 shows how the animation works. Every operation of a given specification is pointed by a control entity. The figure shows a semaphore composed of two operations (master and slave) (Verilog, 1994]. This entity points at the initial symbols of each operation, and then to their subsequent operations, according to given navigation options. 


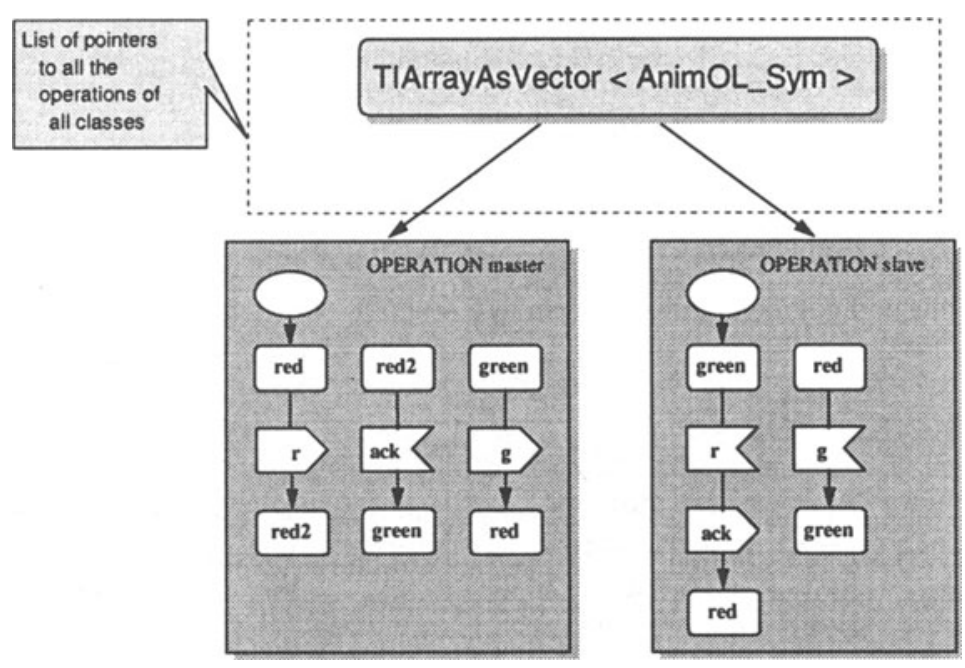

Figure 5 Visiting tree of a given specification

OST's animation provides the following operations: Start, Next Step, Previous Step, Auto, Next Stop and Stats (Eijk, 1988).

1. Start: sets the animation environment and enables the Next Step operation.

2. Next Steps Menu: show actions that can be started. When this menu cannot be enabled, that means, when no operation can offer a transition to be started, the animation will have reached a deadlock state and therefore cannot be run any further. A message will inform the user of any such case.

3. Next Step: allows the start of a new transition in the animation. After the menu is built, the user is expected to interact with the system by indicating which transition should be started.

4. Previous Step: allows reversing one or more steps of the animation. It gives the opportunity to test many execution alternatives, though preventing the need to restart the whole process repeatedly.

5. Auto: runs the animation automatically, with no interaction with the user. Therefore, the system should be informed about the conditions to halt the animation. This conditions are linked to a certain number of iterations over a symbol or to a set run time. Of course, deadlocks will also halt the execution.

6. Next Stop: combines interactive and automatic execution. It allows the animation to be automatized to a point where a decision has to be made. Then, the user will choose which action to take.

7. Stats: these can be checked during or after the animation, presenting information such as:

- Elapsed time: shows elapsed during animation.

- Unvisited symbols: a very useful option, as it shows dead code in the specification. It can be expanded to show whole-unused paths. 
- Potential livelock points: extensively visited points can be thought of as livelocks.

- Total visited symbols: general overview of the total visitation.

The functions available in the animation module enable a larger and clearer view of the specification being developed. Furthermore, the user can assess specific situations and decide if they perform as expected.

\section{CODE GENERATOR}

The language hierarchy provides instruments for automatic code generation (Fröberg, 1993). The classes defined at the abstract structural level are mapped to $\mathrm{C}++$ classes. Each defined class is coded in a separate module, containing the definition of the operations and attributes that are converted, respectively, into functions and variables.

When a class is initialized, each operation is run as a process and its descriptor is stored as an internal attribute to the class, like user-defined attributes. The ports, in their turn, become the methods visible externally to the class. Each method receives messages from external operations to the class and forwards them to their corresponding destiny processes.

The datagram function in figure 6 shows the code generation. As we see, each port is mapped to a method that will be used to provide communication with other classes. Similarly, figure 5.2 shows operation receive receiving a signal $a$. The class's constructor initializes its operations in separate processes. Finally, each operation is defined from a state machine, in non-structured source code.

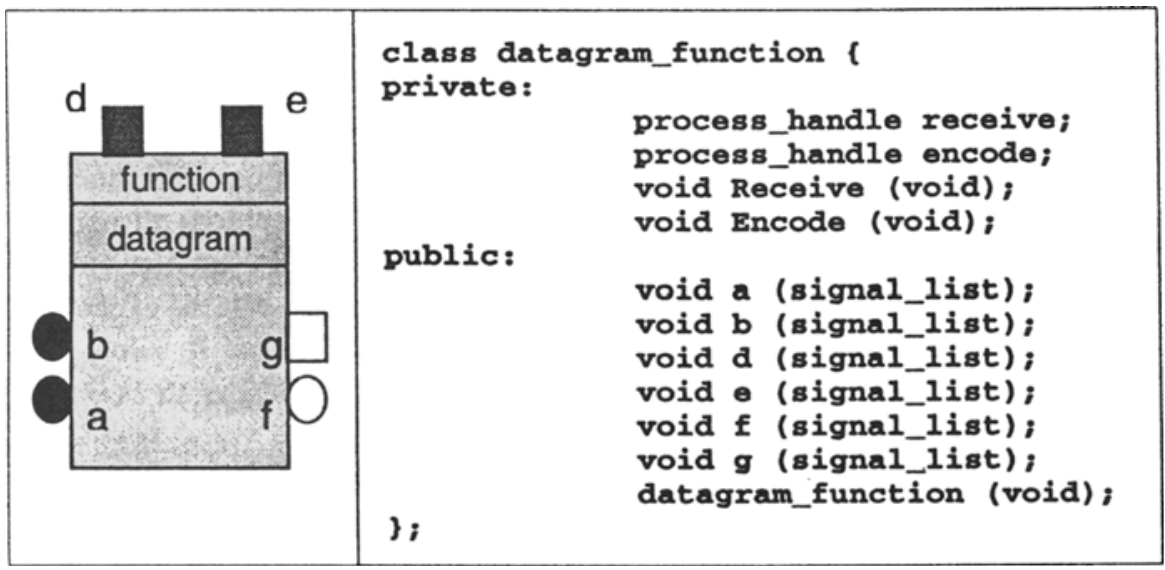

Figure 6 datagram function class 


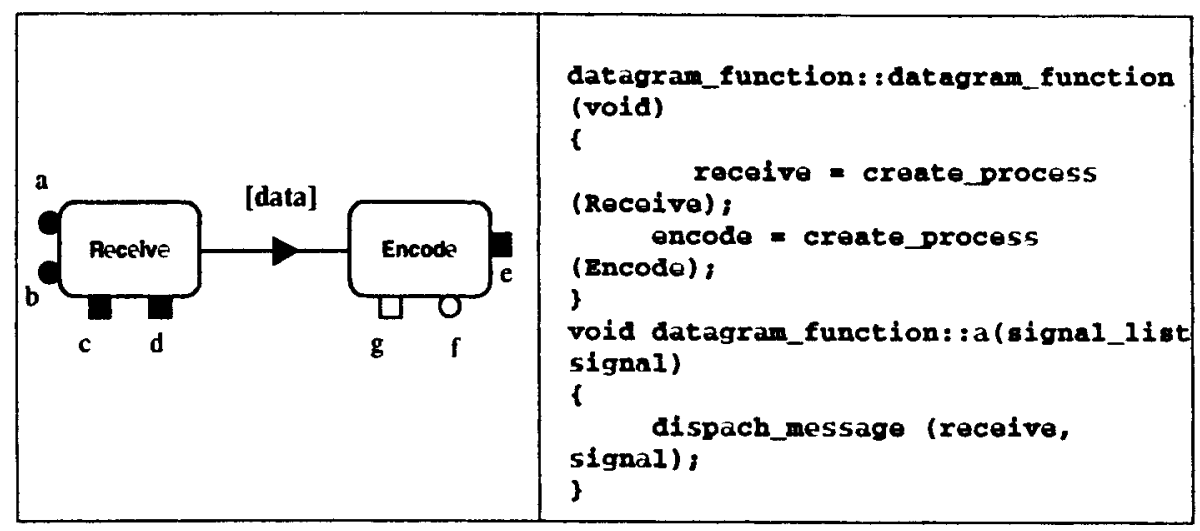

Figure 7 datagram function class's operations

\section{EXAMPLE - THE HTTP PROTOCOL}

The figure 8 shows the diagrams resulting from the HTTP Protocol Specification. It's an incomplete high level description, where details were neglected in order to have a more didactic example.

The protocol is designed as two distinct classes named Server and Client, each of them inheriting the classes corresponding to the HTTP previous version. HTTP first versions define just simple requests, which are less complex than the current one. So, inheriting such definition is a fast way to design more complex specifications.

The client class has an operation named Request, which creates and sends HTTP messages to the server side and waits for the server response. Messages are sent and received via ports (in the example $I n$ and Out). Such ports are showed in the Fine Structural Level diagram and are placed next to the corresponding operation. The behavior of Request is defined in the Operational Level that is quite similar to an ordinary standard SDL diagram. The messages exchanged by classes are done by means of external ports and the associations of them are defined in the External Signals diagram. 




Figure 8 HTTP protocol example diagrams

\section{CONCLUSIONS}

OST is a very powerful specification tool. It provides any users, either computer networks programmers or information systems designers, with a tool based on a standard FDT, such as SDL, and then improves this tool using an object-oriented paradigm, which is known as a potential productivity booster in software development.

The many modules that form OST offer a more efficient way to specify systems. Its editor allows the creation of a specification using NSOMA's graphic grammar, furthering comprehension and interaction. OST's on-line syntax check avoids user errors. In addition, classes can be reused, which makes the construction process ever evolving, incremental, fast and efficient. Under the 
computer networks area jargon, classes can represent the basic concepts of model, layer, protocol, service and entity.

OST's animation feature increases comprehension of the systems developed. This is fundamental for a software developing team where a single and clear idea of what is being done is a must. Also, errors associated to the dynamic behavior of the specification (deadlocks and livelocks, for instance) can be detected. Furthermore, automatic code generation avoids the manual codification of a whole system. Consequently, the source code is free of lexical and syntactical errors.

OST can be further expanded, including specification verification and validation mechanisms that will assure even better correctness. Even though the editor is syntax-oriented, no lexical consistency is performed. This reflects on source code generation, which becomes undesirably linked to errors from the specification stage. Such lexical consistency is also to be included in future expansions.

\section{REFERENCES}

Almeida, Maria Janilce (1994) Especificação de sistemas na área de redes de computadores: uma abordagem orientada a objetos. Porto Alegre: UFRGS (tese de doutorado).

Allende, Jesús Sánchez. (1995) GLAv2.0 - Graphical Animation for LOTOS Quick reference. Technical University of Madrid. Spain.

Cohen, B. et al. (1986) The Specification of Complex Systems. Great Britain: Addyson Wesley.

van Eijk, Peter Herman Johan (1988) Software tools for the specification language LOTOS. Twente University.

Fröberg, M. W. (1993) Automatic Code Generation from SDL to a Declarative Programming Language. Proceedings of SDL Forum.

Gaspary, Luciano Paschoal (1995) Editor gráfico do nível operacional do SDL OO. Porto Alegre: UFRGS (trabalho de diplomação).

Gaspary, Luciano Paschoal (1996) Estudo de Simulação e Animação em Ambientes de Especificação de Redes. PortoAlegre: UFRGS (trabalho individual).

Granville, Lisandro Zambenedetti (1995) Editor gráfico dos níveis estrutural abstrato e estrutural detalhado do SDL OO. Porto Alegre: UFRGS (trabalho de diplomação).

Rumbaugh, James (1991) Object-oriented modeling and design. New Jersey: Prentice-Hall-Englewood Cliffs.

Trainini, Paulo Silveira (1995) Manipulador das estruturas do editor gráfico para SDL OO. Porto Alegre: UFRGS (Trabalho de diplomação).

Verilog Corporation. Geode - Technical Presentation. Preliminary Version. July, 1994. 